\title{
The Existence and Uniqueness of Periodic Solutions for Nonlinear Neutral dynamic equation with infinite delay on a Time Scale.
}

\author{
Amira Ali Ben Fayed *, Mohamed Illafe" ${ }^{* *}$ Haitham A. Makhzoum ${ }^{* * *}$, Rafik.A.Elmansouri ${ }^{* * * *}$ \\ *Department of Mathematics, Faculty of Education, University of Benghazi, Benghazi-Libya \\ ${ }^{* *}$ School of Engineering, Math. \& Technology, Navajo Technical University, NM USA \\ **** Department of mathematic, Faculty of Science, University of Benghazi, Benghazi-Libya \\ ***** College of Electrical and Electronic Technology, Benghazi-Libya \\ DOI: 10.29322/IJSRP.11.10.2021.p11846 \\ http://dx.doi.org/10.29322/IJSRP.11.10.2021.p11846
}

Abstract- This paper aims to prove the existence and uniqueness of a nonlinear neutral dynamic equation with infinite delay using the Krasnoselskii fixed point method and the contraction mapping principle, respectively. Let $\mathbb{T}$ be a periodic time scale; we will consider the following nonlinear neutral dynamic:

$$
x^{\Delta}(t)=-\prod_{i=1}^{p} a_{i}(t) x^{\sigma}(t)+\sum_{i=1}^{p} Q_{i}(t, x(t-g(t)))^{\Delta}+\int_{-\infty}^{t}\left[\prod_{i=1}^{p} D_{i}(t, s) f(x(s))+h(s)\right] \Delta s+G(t, x(t), x(t-\tau(t))), t \in \mathbb{T}
$$

Index Terms- Fixed point, infinite delay, time scales periodic solution.

\section{INTRODUCTION}

$\mathrm{R}$ ecently, researchers have given special attention to the methods concerned with finding a solution to the nonlinear neutral dynamic equation with an infinite delay. These include the fixed-point theorem, Pickard's successive approximation, and the nonexpansive operators' technique. The fixed point method has been declared a powerful method in studying the nonlinear neutral dynamic equation. For a wealth of reference material on the subject, we refer readers to $[1,4,11,12]$ and their references. In addition, fixed-point theory gives the conditions for which maps have solutions, and it has a lot of application in mathematics and other branches of science. Using the Krasnoselskii method, Ben Fayed in [7] studied the existence and uniqueness of solutions for the following nonlinear neutral dynamic equation with infinite delay.

$$
\begin{aligned}
\frac{d}{d t} x(t)=- & \prod_{i=1}^{p} a_{i}(t) x(t)+\frac{d}{d t} \sum_{i=1}^{p} Q_{i}(t, x(t-g(t)))+\int_{-\infty}^{t}\left[\prod_{i=1}^{p} D_{i}(t, s) f(x(s))+h(s)\right] d s \\
& +G(t, x(t), x(t-\tau(t)))
\end{aligned}
$$

In this paper, we will study different nonlinear neutral dynamic equations with an infinite delay for $t \in \mathbb{T}$.

$$
\begin{gathered}
x^{\Delta}(t)=\sum_{i=1}^{p} Q_{i}(t, x(t-g(t)))+\left(1-e_{\ominus a}(t, t-T)\right)^{-1} \times-\left[\int_{t-T}^{t} \prod_{i=1}^{p} a_{i}(t) \sum_{i=1}^{p} Q_{i}^{\sigma}(u, x(u-g(u)))\right] e_{\ominus a}(t, u) \Delta u \\
+\int_{t-T}^{t} \int_{-\infty}^{u}\left[\prod_{i=1}^{p} D_{i}(u, s) f(x(s))+h(s)\right] \Delta s e_{\ominus a}(t, u) \Delta u+\int_{t-T}^{t}[G(u, x(u), x(u-\tau(u)))] e_{\ominus a}(t, u) \Delta u
\end{gathered}
$$

Where $a(t)$ is a function of continuous real value, given that $Q: \mathbb{R} \times \mathbb{R} \rightarrow \mathbb{R}, D: \mathbb{R} \times \mathbb{R} \rightarrow \mathbb{R}, f: \mathbb{R} \rightarrow \mathbb{R}, x: \mathbb{R} \rightarrow \mathbb{R}$ and $h: \mathbb{R} \rightarrow \mathbb{R}$ are Continuous functions. Also, to ensure periodicity, we assumed that $a(t), g(t), D(t, x), Q(t, x)$ are periodic functions.

Our work is relatively different from the work that was done by Ben Fayed [7] when it comes to the equation and the space. We are interested in studying the existence of periodic solutions of Eq (1.2) on a space called the timescale space, which is a relatively new topic that is defined as a closed, nonempty subset of $\mathbb{R}$. The significance of this space is to link the theory of difference equations with the theory of differential equations. See [2,3,5,6,8,9] 
To achieve the desired results, it requires us to satisfy the conditions of Krasnoselskii fixed point theorem, so it was necessary to assume that $z=A z+B z$ provided $z \in M$ where $M$ is a convex set, $A z$ is continuous and compact, $B z$ is a contraction. Also, to fulfill the two mapping conditions of Krasnoselskii's fixed-point theorem, it was necessary to transfer equation (1.2) into an integral equation, which is done in lemma (3.2). Then, we showed that $A z$ is continuous and compact and $B z$ is a contraction. Finally, after satisfying the Krasnoselski theorem condition, we proved the existence of the periodic solutions, and we showed the uniqueness of the periodic solution by using the contraction mapping principle.

\section{PRELIMINARIES}

The primary purpose of this section is to familiarize the reader with any significant notations related to concepts related to the calculus on time scales for dynamic equations. In general, definitions, lemmas, and theorems can be found in $[8,9]$.

A time scale $\mathbb{T}$ is a closed nonempty subset of $\mathbb{R}$. For $t \in T$ the forward jump operator $\sigma$, and the backward jump operator $\rho$, respectively are defined as:

$$
\sigma(t)=\inf \{s \in \mathbb{T}: s>t\} \text { and } \rho(t)=\sup \{s \in \mathbb{T}: s<t\} .
$$

These operators allow elements in the time scale to be classified as follows. We say $t$ is:

$\begin{aligned} \text { i. } & \text { Right scattered if } \sigma(t)>t, \\ \text { ii. } & \text { Right dense if } \sigma(t)=t, \\ \text { iii. } & \text { Left scattered if } \rho(t)<t, \\ \text { iv. } & \text { Left dense if } \rho(t)=t .\end{aligned}$

The graininess function $\mu: \mathbb{T} \rightarrow[0, \infty)$, is defined by $\mu(t)=\sigma(t)-t$ and gives the distance between an element and its successor. We set inf $\varnothing=\sup T$ and $\sup \emptyset=\inf \mathbb{T}$. If $\mathbb{T}$ has a left scattered maximum $\mathrm{M}$, we define $\mathbb{T}^{k}=T \backslash\{M\}$. Otherwise, we define $\mathbb{T}^{k}=T$. If $\mathbb{T}$ has a right scattered minimum $\mathrm{m}$, we define $\mathbb{T}_{k}=T \backslash\{m\}$. Otherwise, we define $\mathbb{T}_{k}=T$.

Let $t \in \mathbb{T}^{k}$ and let $f: \mathbb{T} \rightarrow \mathbb{R}$. The delta derivative of $f(t)$, denoted by $f^{\Delta}(t)$, is defined to be the number (when it exists), with the property that, for each $\epsilon>0$, there is a neighborhood $U$ of $t$ such that

$\left|f(\sigma(t))-f(s)-f^{\Delta}(t)[\sigma(t)-s]\right| \leq \epsilon|\sigma(t)-s|$,

for all $s \in U$. If $\mathbb{T}=\mathbb{R}$ then $f^{\Delta}(t)=\grave{f}(t)$ is the usual derivative. If $\mathbb{T}=\mathbb{Z}$, then $f^{\Delta}(t)=\Delta f(t)=f(t+1)-f(t)$ is the forward difference of $f$ at $t$.

A function $f$ is right dense continuous (rd-continuous), $f \in C_{r d}=C_{r d}(\mathbb{T}, \mathbb{R}$ ), if it is continuous at every right dense point $t \in \mathbb{T}$ and its left-hand limits exist at each left dense point $t \in \mathbb{T}$. function $f: \mathbb{T} \rightarrow \mathbb{R}$. is differentiable on $\mathbb{T}^{k}$ provided $f^{\Delta}(t)$ exists for all $t \in \mathbb{T}^{k}$. We are now able to state some properties of the delta-derivative of $f$. Note that $f^{\sigma}(t)=f(\sigma(t))$.

Theorem 2.1. [8]. Assume that $f, g: \mathbb{T} \rightarrow \mathbb{R}$ are differentiable at $t \in \mathbb{T}^{k}$ and let $\alpha$ be a scalar.

$$
\begin{aligned}
\text { i. } & (f+g)^{\Delta}(t)=f^{\Delta}(t)+g^{\Delta}(t) . \\
\text { ii. } & (\alpha f)^{\Delta}(t)=\alpha f^{\Delta}(t) . \\
\text { iii. } & (f g)^{\Delta}(t)=f^{\Delta}(t) g(t)+f^{\sigma}(t) g^{\Delta}(t) . \\
\text { iv. } & (f g)^{\Delta}(t)=f(t) g^{\Delta}(t)+f^{\Delta}(t) g^{\sigma}(t) . \quad \text { (The product rules) } \\
\text { v. } & \text { If } g(t) g^{\sigma}(t) \neq 0 \text { then } \\
& \\
& \left(\frac{f}{g}\right)^{\Delta}(t)=\frac{f^{\Delta}(t) g(t)-f(t) g^{\Delta}(t)}{g(t) g^{\sigma}(t)} .
\end{aligned}
$$

The first two theorems deal with the composition of two functions. The first theorem is the chain rule on time scales[8] .

Theorem 2.2 (Chain Rule). Assume, $v: \mathbb{T} \rightarrow \mathbb{R}$ is strictly increasing and $\widetilde{\mathbb{T}}:=v(\mathbb{T})$ is a time scale.

Let $w: \widetilde{\mathbb{T}} \rightarrow \mathbb{R}$. If $v^{\Delta}(t)$ and $w^{\grave{\Delta}}(v(t))$ exist for $t \in \mathbb{T}^{k}$, then $(w \circ v)^{\Delta}=\left(w^{\dot{\Delta}} \circ v\right) v^{\Delta}$.

In the sequel, we will need to differentiate and integrate functions of the form $f(t-g(t))=f(v(t))$, where $v(t):=t-g(t)$. The second theorem is the substitution rule[8].

Theorem 2.3 (Substitution). Assume $v: \mathbb{T} \rightarrow \mathbb{R}$ is strictly increasing and $\widetilde{\mathbb{T}}=v(\mathbb{T})$ is a time scale. If $f: \mathbb{T} \rightarrow \mathbb{R}$ is an rd-continuous function and $v$ is differentiable with rd-continuous derivative, then for $a, b \in \mathbb{T}$, 


$$
\int_{a}^{b} f(t) v^{\Delta}(t) \Delta t=\int_{v(a)}^{v(b)}\left(f \circ v^{-1}\right)(s) \tilde{\Delta} \mathrm{s}
$$

A function $p: \mathbb{T} \rightarrow \mathbb{R}$ is said to be regressive provided $1+\mu(t) p(t) \neq 0$ for all $t \in \mathbb{T}^{k}$. The set of all regressive rd-continuous functions $f: \mathbb{T} \rightarrow \mathbb{R}$ is denoted by $\mathcal{R}$ while the set $\mathcal{R}^{+}$is given by

$$
\mathcal{R}^{+}=\{f \in \mathcal{R}: 1+\mu(t) f(t)>0 \text { for all } t \in \mathbb{T}\} .
$$

Let $p \in \mathcal{R}$ and $\mu(t) \neq 0$ for all $t \in \mathbb{T}$. The exponential function on $\mathbb{T}$ is defined by

$$
e_{p}(t, s)=\exp \left(\int_{s}^{t} \frac{1}{\mu(z)} \log (1+\mu(z) p(z)) \Delta z\right) .
$$

It is well known that if $p \in \mathcal{R}^{+}$, then $e_{p}(t, s)>0$ for all $t \in \mathbb{T}$. Also, the exponential function $y(t)=e_{p}(t, s)$ is the solution to the initial value problem $y^{\Delta}=p(t) y, y(s)=1$. Other properties of the exponential function are given in the following lemma, [8].

Lemma 2.4. Let $p, q \in \mathcal{R}$. Then

$$
\begin{aligned}
\text { i. } & e_{0}(t, s)=1 \text { and } e_{p}(t, t)=1, \\
\text { ii. } & e_{p}(\sigma(t), s)=(1+\mu(t) p(t)) e_{p}(t, s), \\
\text { iii. } & \frac{1}{e_{p}(t, s)}=e_{\ominus p}(t, s), \text { where } \ominus p(t)=-\frac{p(t)}{1+\mu(t) p(t)}, \\
\text { iv. } & e_{p}(t, s)=\frac{1}{e_{p}(s, t)}=e_{\ominus p}(s, t), \\
\text { v. } & e_{p}(t, s) e_{p}(s, r)=e_{p}(t, r), \\
\text { vi. } & \left(\frac{1}{e_{p}(., s)}\right)^{\Delta}=-\frac{p(t)}{e_{p}^{\sigma}(., s)} .
\end{aligned}
$$

The notion of periodic time scales and the following two definitions are quoted from [8] and[11].

Definition 2.5. We say that a time scale $\mathbb{T}$ is periodic if there exists $p>0$, such that, if $t \in \mathbb{T}$, then $t \pm p \in \mathbb{T}$. For $\mathbb{T} \neq \mathbb{R}$, the smallest positive $p$ with this property is called the period of the time scale.

Example 2.6. The following time scales are periodic.

1. $\mathbb{T}=\bigcup_{i=-\infty}^{\infty}[2(i-1) h, 2 i h], h>0$ has provided $p=2 h$.

2. $\mathbb{T}=h Z$ has period $p=h$.

3. $\mathbb{T}=\mathbb{R}$

4. $\mathbb{T}=\left\{t=k-q^{m}: k \in z, m \in N_{0}\right\}$, where $0<q<1$ has period $p=1$.

Remark 2.7 [11]. All periodic time scales are unbounded above and below.

Definition 2.8. Let $\mathbb{T} \neq \mathbb{R}$ be a periodic time scale with period $T$. We say that the function $f: \mathbb{T} \rightarrow \mathbb{R}$ is periodic with period $T$ if there exists a natural number $n$ such that $T=n p, f(t \pm T)=f(t)$ for all $t \in \mathbb{T}$ and $T$ is the smallest number such that $f(t \pm T)=f(t)$.

If $\mathbb{T}=\mathbb{R}$, we say that $f$ is periodic with period $T>0$ if $T$ is the smallest positive number such that $f(t \pm T)=f(t)$ for all $t \in \mathbb{T}$.

Remark 2.9 [11]. If $\mathbb{T}$ is a periodic time scale with period $p$, then $\sigma(t \pm n p)=\sigma(t) \pm n p$. Consequently, the graininess function $\mu$ satisfies $\mu(t \pm n p)=\sigma(t \pm n p)-(t \pm n p)=\sigma(t)-t=\mu(t)$, and so it is a periodic function with period $p$.

\section{EXISTENCE OF PERIODIC SOLUTIONS}

In this section, we will present the main result. All steps in this work are inspired and motivated by the works done by Makhzoum $[13,14]$. The following conditions should be assumed. Let $C(\mathbb{T}, \mathbb{R})$ be the space of all real-valued continuous functions on $\mathbb{T}$. Define

$$
\mathcal{H}_{T}=\{\varphi \in C(\mathbb{T}, \mathbb{R}): \varphi(t+T)=\varphi(t)\}, \text { where } T>0 ; T \in \mathbb{T} .
$$

then $\mathcal{H}_{T}$ is a Banach space with the supremum norm

$$
\|x\|=\sup |x(t)|, \quad t \in[0, T] .
$$

If $\mathbb{T} \neq \mathbb{R}, T=n p$ for some, $n \in \mathbb{N}$. By the notation $[a, b]$ we mean

$$
[a, b]=\{t \in \mathbb{T}: a \leq t \leq b\}
$$


unless otherwise specified. The intervals $[a, b),(a, b]$, and $(a, b)$ are defined similarly. For all $t \in \mathbb{T}$, let $a(t)>0$ and $a \in \mathcal{R}^{+}$, where $a(t)$ is continuous, and

$$
a(t+T)=a(t), g(t+T)=g(t), D(t+T, x)=D(t, x), G(t+T, x, y)=G(t, x, y)
$$

Where $i d$ is the identity function on $\mathbb{T}$, we also assume that $Q(t, x)$ and $f(x)$ are continuous and periodic in $t$ and Lipschitz continuous in $x$. That is,

$$
Q(t+T, x)=Q(t, x)
$$

and there are positive constants $E_{1}, E_{2}, E_{3}, E_{4}, E_{5}$ and $E_{6}$ such that

and

$$
\int_{-\infty}^{t} \prod_{i=1}^{p}\left|D_{i}(t, s)\right| d s \leq E_{3}<\infty, h(s) \leq E_{4}
$$

Lemma 3.1. [11]. Let $x \in \mathcal{H}_{T}$. Then $\left\|x^{\sigma}\right\|$ exists and $\left\|x^{\sigma}\right\|=\|x\|$.

The following lemma is used to covert the equation (1.2) to an equivalent integral equation,

Lemma 3.2. Suppose (3.1), (3.2) hold, if $x \in \mathcal{H}_{T}$, then $x$ is a solution of Eq (1.2) if and only if

$$
\begin{aligned}
x(t) & =\sum_{i=1}^{p} Q_{i}(t, x(t-g(t)))+\left(1-e_{\ominus a}(t, t-T)\right)^{-1} \times-\left[\int_{t-T}^{t} \prod_{i=1}^{p} a_{i}(t) \sum_{i=1}^{p} Q_{i}^{\sigma}(u, x(u-g(u)))\right] e_{\ominus a}(t, u) \Delta u \\
& +\int_{t-T}^{t} \int_{-\infty}^{u}\left[\prod_{i=1}^{p} D_{i}(u, s) f(x(s))+h(s)\right] \Delta s e_{\ominus a}(t, u) \Delta u+\int_{t-T}^{t}[G(u, x(u), x(u-\tau(u)))] e_{\ominus a}(t, u) \Delta u
\end{aligned}
$$

Proof. Let $x(t) \in \mathcal{H}_{T}$ be a solution of Eq (1.1). By writing Eq (1.1) as

$$
\left[x(t)-\sum_{i=1}^{p} Q_{i}(t, x(t-g(t)))\right]^{\Delta}=-\prod_{i=1}^{p} a_{i}(t) x(t)+\int_{-\infty}^{t}\left[\prod_{i=1}^{p} D_{i}(t, s) f(x(s))+h(s)\right] \Delta s+G(t, x(t), x(t-\tau(t)))
$$

Adding $\prod_{i=1}^{p} a_{i}(t) \sum_{i=1}^{p} Q_{i}^{\sigma}(t, x(t-g(t)))$ to both sides of the equation (3.7), we find:

$$
\begin{gathered}
{\left[x(t)-\sum_{i=1}^{p} Q_{i}(t, x(t-g(t)))\right]^{\Delta}=-\prod_{i=1}^{p} a_{i}(t)\left[x^{\sigma}(t)-\sum_{i=1}^{p} Q_{i}^{\sigma}(t, x(t-g(t)))\right]} \\
-\prod_{i=1}^{p} a_{i}(t) \sum_{i=1}^{p} Q_{i}^{\sigma}(t, x(t-g(t)))+\int_{-\infty}^{t}\left[\prod_{i=1}^{p^{p}} D_{i}(t, s) f(x(s))+h(s)\right] \Delta s+G(t, x(t), x(t-\tau(t)))
\end{gathered}
$$

Multiply both sides of $(2.8)$ by $e_{a}(t, 0)$, then integrate from $t-T$ to $t$, we have

$$
\begin{gathered}
{\left[x(t)-\sum_{i=1}^{p} Q_{i}(t, x(t-g(t)))\right] e_{a}(t, 0)-\left[x(t-T)-\sum_{i=1}^{p} Q_{i}(t-T, x(t-T-g(t-T)))\right] e_{a}(t-T, 0)} \\
=\int_{t-T}^{t}\left[-\prod_{i=1}^{p} a_{i}(u) \sum_{i=1}^{p} Q_{i}^{\sigma}(u, x(u-g(u)))+\int_{-\infty}^{u}\left[\prod_{i=1}^{p} D_{i}(u, s) f(x(s))+h(s)\right] \Delta s\right. \\
+G(u, x(u), x(u-\tau(u)))] e_{a}(u, 0) \Delta u
\end{gathered}
$$

By dividing both sides of the above equation by $e_{a}(t, 0)$ and knowing that $x(t)$ is a periodic function of period $T$ and using Eq (3.1) and $\mathrm{Eq}$ (3.2), we get:

$x(t)=\sum_{i=1}^{p} Q_{i}(t, x(t-g(t)))+\left(1-e_{\ominus a}(t, t-T)\right)^{-1} \times\left[-\int_{t-T}^{t} \prod_{i=1}^{p} a_{i}(u) \sum_{i=1}^{p} Q_{i}^{\sigma}(u, x(u-g(u)))\right] e_{\theta a}(t, u) \Delta u+$ $\int_{t-T}^{t} \int_{-\infty}^{u}\left[\prod_{i=1}^{p} D_{i}(u, s) f(x(s))+h(s)\right] \Delta s e_{\theta a}(t, u) \Delta u+\int_{t-T}^{t}[G(u, x(u), x(u-\tau(u)))] e_{\theta a}(t, u) \Delta u$, 
the proof is complete.

We will introduce the statement of Krasnoselskii's fixed-point theorem and apply this theorem to prove the existence of a periodic solution.

Theorem 3.3 (Krasnoselskii). Let Mbe a closed convex nonempty subset of a Banach space ( $B$, $\|$. \|) Suppose that $A$ and $B$ map $\mathbb{M}$ into $\mathbb{B}$ such that

(i) $x, y \in \mathbb{M}$, implies $A x+B y \in \mathbb{M}$

(ii) $A$ is compact and continuous,

(iii) $B$ is a contraction mapping.

Then there exists $z \in \mathbb{M}$ with $z=A z+B z$.

For its proof, we refer the reader to [15]. As stated on the hypothesis of theorem 3.3, there are two mappings, one is a contraction, and the other is compact. Therefore, we will define the mapping $P: \mathcal{H}_{T} \rightarrow \mathcal{H}_{T}$ by

$$
\begin{aligned}
(P \varphi)(x)=\sum_{i=1}^{p} Q_{i}(t, \varphi(t-g(t)))+\left(1-e_{\ominus a}(t, t-T)\right)^{-1} \times\left[-\int_{t-T}^{t} \prod_{i=1}^{p} a_{i}(u) \sum_{i=1}^{p} Q_{i}^{\sigma}(u, \varphi(u-g(u)))\right] e_{\theta a}(t, s) \Delta u \\
\quad+\int_{t-T}^{t} \int_{-\infty}^{u}\left[\prod_{i=1}^{p} D_{i}(u, s) f(\varphi(s))+h(s)\right] \Delta s e_{\theta a}(t, s) \Delta u+\int_{t-T}^{t}[G(u, \varphi(u), x(u-\tau(u)))] e_{\theta a}(t, s) \Delta u
\end{aligned}
$$

By using the same steps in [1], we can prove that, $(P \varphi)(x)$ is periodic in $t$ of period $T$.

Now by expressing equation (3.9) as

where $A$ and $B$ are given by

$$
(P \varphi)(t)=(B \varphi)(t)+(A \varphi)(t)
$$

and,

$$
(B \varphi)(t)=\sum_{i=1}^{p} Q_{i}(t, \varphi(t-g(t)))
$$

$$
\begin{aligned}
(A \varphi)(t)=(1- & \left.e_{\ominus a}(t, t-T)\right)^{-1}\left[-\int_{t-T}^{t} \prod_{i=1}^{p} a_{i}(u) \sum_{i=1}^{p} Q_{i}^{\sigma}(u, \varphi(u-g(u)))\right] e_{\theta a}(t, s) \Delta u \\
& +\int_{t-T}^{t} \int_{-\infty}^{u}\left[\prod_{i=1}^{p} D_{i}(u, s) f(\varphi(s))+h(s)\right] \Delta s e_{\theta a}(t, s) \Delta u+\int_{t-T}^{t}[G(u, \varphi(u), x(u-\tau(u)))] e_{\theta a}(t, s) \Delta u
\end{aligned}
$$

We are trying to achieve that $(B \varphi)(t)$ is contraction and $(A \varphi)(t)$ is compact this can be done by providing these two lemmas, but before introducing the lemmas, we will define the following constants

$$
\begin{aligned}
\tau & :=\max _{t \in[0, T]}\left|\left(1-e_{\theta a}(t, t-T)\right)^{-1}\right|, \\
v & :=\max _{u \in[t-T, t]}\left|e_{\theta a}(t, u)\right|, \\
\rho & :=\max _{t \in[0, T]} \prod_{i=1}\left|a_{i}(t)\right| .
\end{aligned}
$$

Lemma 3.4. If $A$ is defined by (3.11), then $A$ is continuous, and the image of $A$ is contained in a compact set.

Proof. We will start by proving $A$ is continuous; we define $A$ as in (3.11). Let $\varphi, \psi \in \mathcal{H}_{T}$, for a given $\varepsilon>0$, take $\delta=\frac{\varepsilon}{\mathrm{N}}$ with $N=$ $\tau v T\left[\rho E_{1}+E_{3} E_{2}+E_{4}+E_{5}+E_{6}\right]$, now for $\|\varphi-\psi\|<\delta$, and by using (3.3) into (3.6), we get:

$$
\left\|A_{\varphi}-A_{\psi}\right\| \leq \tau \nu T\left[\rho E_{1}+E_{3} E_{2}+E_{4}+E_{5}+E_{6}\right]\|\varphi-\psi\| \leq \mathrm{N}\|\varphi-\psi\| \leq \mathrm{N} \delta \leq \varepsilon .
$$

This shows that $A$ is continuous. Now, we need to show that $A$ is a compact set. By using Ascoli-Arzela's theorem [10] which states that for $A \subset X, A$ is compact if and only if $A$ is bounded and equi-continuous.

Let $\Omega=\left\{\varphi \in \mathcal{H}_{T}:\|\varphi\| \leq \Upsilon\right\}$, where $\Upsilon$ is any fixed positive constant, from (3.3) and (3.4) we have, 


$$
\begin{aligned}
\sum_{i=1}^{p}\left|Q_{i}(t, x)\right| & =\sum_{i=1}^{p}\left|Q_{i}(t, x)-Q_{i}(t, 0)+Q_{i}(t, 0)\right| \\
& \leq \sum_{i=1}^{p}\left[\left|Q_{i}(t, x)-Q_{i}(t, 0)\right|+\left|Q_{i}(t, 0)\right|\right] \\
& \leq E_{1}\|x\|+\alpha,
\end{aligned}
$$

Where $\alpha=\sup _{t \in[0, T]} \sum_{i=1}^{p}\left|Q_{i}(t, 0)\right|$.

Similarly,

and,

$$
|f(x)|=|f(x)-f(0)| \leq|f(x)-f(0)| \leq E_{2}\|x\| .
$$

$$
\begin{aligned}
|G(t, x, y)| & =|G(t, x, y)-G(t, 0,0)+G(t, 0,0)| \\
& \leq|G(t, x, y)-G(t, 0,0)|+|G(t, 0,0)| \\
& \leq E_{5}\|x\|+E_{6}\|y\|
\end{aligned}
$$

Taking into consideration, $f(0)=0$ and $G(t, 0,0)=0$. Let $\varphi_{n} \in \Omega$ where $n$ is a positive integer with $L=\tau v T\left[\rho E_{1}(\gamma+\alpha)+\right.$ $\left.E_{3} E_{2} \Upsilon+E_{4}+\Upsilon\left(E_{5}+E_{6}\right)\right]$ where $L>0$, Therefore,

$$
\begin{gathered}
\left\|A_{\varphi_{n}}\right\|=\mid\left(1-e_{\ominus a}(t, t-T)\right)^{-1}\left[-\int_{t-T}^{t} \prod_{i=1}^{p} a_{i}(u) \sum_{i=1}^{p} Q_{i}^{\sigma}\left(u, \varphi_{n}(u-g(u))\right)\right] e_{\theta a}(t, s) \Delta u \\
+\int_{t-T}^{t} \int_{-\infty}^{u}\left[\prod_{i=1}^{p} D_{i}(u, s) f\left(\varphi_{n}(s)\right)+h(s)\right] \Delta s e_{\theta a}(t, s) \Delta u+\int_{t-T}^{t}\left[G\left(u, \varphi_{n}(u), \varphi_{n}(u-\tau(u))\right)\right] e_{\theta a}(t, s) \Delta u \mid \\
\leq \max _{t \in[0, T]} \mid\left(1-e_{\ominus a}(t, t-T)\right)^{-1}\left[-\int_{t-T}^{t} \prod_{i=1}^{p} a_{i}(u) \sum_{i=1}^{p} Q_{i}^{\sigma}\left(u, \varphi_{n}(u-g(u))\right)\right] e_{\theta a}(t, s) \Delta u \\
+\int_{t-T}^{t} \int_{-\infty}^{u}\left[\prod_{i=1}^{p} D_{i}(u, s) f\left(\varphi_{n}(s)\right)+h(s)\right] \Delta s e_{\theta a}(t, s) \Delta u+\int_{t-T}^{t}\left[G\left(u, \varphi_{n}(u), \varphi_{n}(u-\tau(u))\right)\right] e_{\theta a}(t, s) \Delta u \mid \\
\leq \tau v\left[-\int_{t-T}^{t}\left[\begin{array}{c}
\left.\rho\left(E_{1}\left\|\varphi_{n}\right\|+\alpha\right)+\int_{-\infty}^{u}\left[\left|\prod_{i=1}^{p} D_{i}(u, s)\right|\left|f\left(\varphi_{n}(s)\right)\right|+|h(s)| \Delta s+E_{5}\left\|\varphi_{n}\right\|+E_{6}\left\|\varphi_{n}\right\|\right] \Delta u\right] \\
\leq \tau v\left[-\int_{t-T}^{t}\left[\rho E_{1}\left(\left\|\varphi_{n}\right\|+\propto\right)+E_{3} E_{2}\left\|\varphi_{n}\right\|+E_{4}+\left\|\varphi_{n}\right\|\left(E_{5}+E_{6}\right)\right] \Delta u\right] \\
\left.\leq \tau v T \rho E_{1}\left(\left\|\varphi_{n}\right\|+\propto\right)+E_{3} E_{2}\left\|\varphi_{n}\right\|+E_{4}+\left\|\varphi_{n}\right\|\left(E_{5}+E_{6}\right)\right] \\
\leq \tau v T\left[\rho E_{1}(Y+\alpha)+E_{3} E_{2} Y+E_{4}+\Upsilon\left(E_{5}+E_{6}\right)\right] \leq L
\end{array}\right.\right.
\end{gathered}
$$

This shows that $A$ is bounded. To prove A is equi-continuous, we need to find $\left(A \varphi_{n}\right)^{\Delta}(t)$ and prove that it is uniformly bounded. Therefore, after derivative (3.11) with using (3.3) - (3.6) we get,

$$
\begin{aligned}
\left(A \varphi_{n}\right)^{\Delta}(t)=- & \prod_{i=1}^{p} a_{i}(t) A\left(\varphi_{n}\right)^{\sigma}(t)-\prod_{i=1}^{p} a_{i}(t) \sum_{i=1}^{p} Q_{i}^{\sigma}\left(t, \varphi_{n}(t-g(t))\right) \\
& +\int_{-\infty}^{t}\left(\prod_{i=1}^{p} D_{i}(t, s) f\left(\varphi_{n}(s)\right)+h(s)\right) \Delta s+G\left(t, \varphi_{n}(t), \varphi_{n}(t-\tau(t))\right)
\end{aligned}
$$

The above expression yields $\left\|\left(A \varphi_{n}\right)^{\Delta}\right\| \leq Z$ where $Z$ is some positive constant. Hence, by Ascoli-Arzela's theorem, $A \varphi$ is compact.

Lemma 3.5. If $B$ is given by (3.10) with $E_{1}<1$, and (3.3) hold, then $B$ is a contraction.

Proof. Let $B$ be defined by (3.10). Then for $\varphi, \psi \in \mathcal{H}_{T}$ we have

By using (3.3), we have

$$
\begin{aligned}
\|(B \varphi)(t)-(B \psi)(t)\| & =\sup _{t \in[0, T]}|(B \varphi)(t)-(B \psi)(t)| \\
& =\sup _{t \in[0, T]} \sum_{i=1}^{p}\left|Q_{i}(t, \varphi(t-g(t)))-Q_{i}(t, \psi(t-g(t)))\right| .
\end{aligned}
$$




$$
\|(B \varphi)(t)-(B \psi)(t)\| \leq E_{1} \sup _{t \in[0, T]}\|\varphi(t-g(t))-\psi(t-g(t))\|
$$

As $E_{1}<1$ therefore, $B$ defines a contraction.

Theorem 3.6. Suppose (3.1) - (3.6) hold. Let $\alpha=\sup _{t[[0, T]} \sum_{i=1}^{p}\left|Q_{i}(t, 0)\right|$, and let $\mathcal{K}$ be a positive constant satisfying the inequality $\tau \nu T\left[\rho\left(E_{1} \mathcal{K}+\alpha\right)+E_{3} E_{2} \mathcal{K}+E_{4}+\mathcal{K}\left(E_{5}+E_{6}\right)\right]+E_{1} \mathcal{K}+\alpha \leq \mathcal{K}$

Let $\mathcal{M}=\left\{\varphi \in \mathcal{H}_{T}:\|\varphi\| \leq \mathcal{K}\right\}$ Then Eq (1.2) has a solution in $\mathcal{M}$.

Proof. First, we will define $\mathcal{M}=\left\{\varphi \in \mathcal{H}_{T}:\|\varphi\| \leq \mathcal{K}\right\}$. Knowing that $\mathrm{A}$ is continuous and $A M$ is contained in a compact set from lemma (3.4). Also, the mapping $B$ is a contraction from lemma (3.5). It is clear that $A, B: \mathcal{H}_{T} \rightarrow \mathcal{H}_{T}$. The aim is to show that $\left\|A_{\varphi}+B_{\psi}\right\| \leq \mathcal{K}$. Let $\varphi, \psi \in \mathcal{M}$, with $\|\varphi\|,\|\psi\| \leq \mathcal{K}$. Then,

Lemma 3.4 says that,

$$
\left\|A_{\varphi}+B_{\psi}\right\| \leq\left\|A_{\varphi}\right\|+\left\|B_{\psi}\right\|
$$

Therefore,

$$
\left\|A_{\varphi_{n}}\right\| \leq \tau v T\left[\rho E_{1}\left(\left\|\varphi_{n}\right\|+\propto\right)+E_{3} E_{2}\left\|\varphi_{n}\right\|+E_{4}+\left\|\varphi_{n}\right\|\left(E_{5}+E_{6}\right)\right]
$$

$$
\begin{aligned}
\|A\|+\|B\| & \leq \tau v T\left[\rho E_{1}\left(\left\|\varphi_{n}\right\|+\propto\right)+E_{3} E_{2}\left\|\varphi_{n}\right\|+E_{4}+\left\|\varphi_{n}\right\|\left(E_{5}+E_{6}\right)\right]+E_{1}\|\psi\|+\alpha \\
& \leq \tau v T\left[\rho E_{1}(\mathcal{K}+\propto)+E_{3} E_{2} \mathcal{K}+E_{4}+\mathcal{K}\left(E_{5}+E_{6}\right)\right]+E_{1} \mathcal{K}+\alpha \leq \mathcal{K} .
\end{aligned}
$$

Hence, all conditions of Theorem 3.3 are proven. Thus, there exists a fixed point $z$ in $\mathcal{M}$. By Lemma 3.2, this fixed point is a solution of $\mathrm{Eq}$ (1.2). Therefore, Eq (1.2) has a T-periodic solution

\section{THE UNIQUENESS OF THE SOLUTION}

Theorem 4.1. Let (3.1) - (3.6) hold if

Then Eq (1.2) has a unique T-periodic solution.

$$
E_{1}+\tau v T\left(\rho E_{1}+E_{3} E_{2}+E_{4}+E_{5}+E_{6}\right)<1
$$

Proof. Let $\varphi, \psi \in \mathcal{H}_{T}$. We define $P$ as Eq (3.9). We have,

$$
\left\|P_{\varphi-} P_{\psi}\right\|<\left[E_{1}+\tau v T\left(\rho E_{1}+E_{3} E_{2}+E_{4}+E_{5}+E_{6}\right)\right]\|\varphi-\psi\| .
$$

This completes the proof of Theorem 4.1.

\section{CONCLUSION}

In this study, we have achieved existence and uniqueness by converting Eq (1.2) into an integral equation that helps us create two mappings: a contraction and a completely continuous. Then, in 3.6 and 4.1, we used theorem 3.3 to show the existence of periodic solutions and the contraction mapping principle to show the uniqueness of the periodic solution.

\section{ACKNOWLEDGMENT}

The authors would like to thank the referees for reading the manuscript, correcting errors, and valuable hints.

\section{REFERENCES}

[1] Althubiti, S., Makhzoum, H. A., \& Raffoul, Y. N. (2013). Periodic solution and stability in nonlinear neutral system with infinite delay. Appl. Math. Sci, 7(136), 6749-6764.

[2] Ardjouni, A., \& Djoudi, A. (2016). Periodicity and Stability in Nonlinear Neutral Dynamic Equations with Infinite Delay on a Time Scale. Khayyam Journal of Mathematics, 2(1), 51-62.

[3] Ardjouni, A., \& Djoudi, A. (2012). Existence of periodic solutions for nonlinear neutral dynamic equations with variable delay on a time scale. Communications in Nonlinear Science and Numerical Simulation, 17(7), 3061-3069.

[4] Ardjouni, A., \& Djoudi, A. (2012). Existence of positive periodic solutions for a nonlinear neutral differential equation with variable delay. Applied Mathematics E-Notes, 12, 94-101.

[5] Ardjouni, A., \& Djoudi, A. (2010). Periodic solutions in totally nonlinear dynamic equations with functional delay on a time scale. Rend. Sem. Mat. Univ. Politec. Torino, 68(4), 349-359.

[6] Atici, F. M., Guseinov, G. S., \& Kaymakcalan, B. (1999). Stability criteria for dynamic equations on time scales with periodic coefficients. In Proceedings of the International Conference on Dynamic Systems and Applications, III (Vol. 3, pp. 43-48).

[7] Ben Fayed A., Alfrgany S., \& Makhzoum H. (2020). The existence and uniqueness of periodic solutions for nonlinear neutral first order differential equation with functional delay. Science Journal of Faculty of Education, 2(8), 214-123. 
[8] Bohner, M., \& Peterson, A. (2001). Dynamic equations on time scales: An introduction with applications. Springer Science \& Business Media.

[9] Bohner, M., \& Peterson, A. C. (Eds.). (2002). Advances in dynamic equations on time scales. Springer Science \& Business Media.

[10] DiBenedetto, E., \& Debenedetto, E. (2002). Real analysis. Boston: Birkhäuser

[11] Kaufmann, E. R., \& Raffoul, Y. N. (2006). Periodic solutions for a neutral nonlinear dynamical equation on a time scale. Journal of Mathematical Analysis and Applications, 319(1), 315-325.

[12] Makhzoum, H., \& Elmansouri R. (2018). The existence and uniqueness of periodic solutions for nonlinear neutral first order differential equation with functional delay. Libyan Journal of Science \& Technology, 7(2), 114-117.

[13] Makhzoum, H., Elmabrok, A., \& Elmansouri, R. (2021). Existence and uniqueness of solutions for neutral periodic integral differential equations with infinite delay on time scale. Caspian Journal of Mathematical Sciences (CJMS), $10(1), 93-103$.

[14] Makhzoum, H., Jalgaf, H., \& Imhemed, A. (2021). The existence and uniqueness of periodic solutions for nonlinear neutral dynamic equations with infinite delay on a Time Scale. Journal of Faculty Education, 9, 221-238.

[15] Smart, D. R. (1980). Fixed point theorems (Vol. 66). Cup Archive.

\section{AUTHORS}

First Author - Amira Ali Ben Fayed, MS of Mathematics, University of Benghazi amira.fayed@uob.edu.ly.

Second Author - Mohamed Illafe, MS and MA of Mathematics, Navajo Technical University millafe@navajotech.edu.

Third Author - Haitham A.Makzoum, MS of Mathematics, University of Benghazi haitham.makhzoum@uob.edu.ly.

Fourth Author - Rafik.A.Elmansouri, MS of Mathematics, College of Electrical and Electronic Technology rafik-

almansouri@ceet.edu.ly.

Correspondence Author-Mohamed Illafe, millafe@navajotech.edu, moillafe@gmail.com, +1575-639-2469. 